\title{
EDITORIAL
}

(cc) BY-NC

\section{The respiratory subtype of panic disorder: reflections on the past and future of biological psychiatry}

\author{
Dan J. Stein iD \\ UCT Department of Psychiatry, Groote Schuur Hospital J2, Cape Town, South Africa.
}

Publication of a paper reviewing the respiratory subtype of panic disorder in the Brazilian Journal of Psychiatry is timely, given the recent passing of Donald Klein, who, among his multiple seminal contributions, initiated work in this area. Don inspired many researchers to follow in his footsteps, amongst them Antonio Nardi's Brazilian group, which authored this paper, and Jack Gorman's group at Columbia University, which pursued related questions.

As this review indicates, there is much to admire about work on the respiratory subtype of panic disorder.

First, this work builds on a tradition of careful clinical observation. Klein's first hypothesis about panic disorder subtypes emerged from his work at Hillside Hospital. On the basis of close and comprehensive examinations, he suggested that one subtype of panic disorder was characterized by early separation anxiety, with panic attacks triggered by separation or bereavement. At Columbia, his ideas developed to emphasize links between panic attacks and the respiratory system.

Second, this work has given impetus to rigorous preclinical and clinical research on causal mechanisms. In the clinical laboratory, $\mathrm{CO}_{2}$ inhalation and other "challenges" were used to induce panic attacks; an early paper by Gorman et al. on the neuroanatomy of panic remains a classic synthesis of biological and psychological findings. ${ }^{2}$ In the animal laboratory, remarkably, evidence has been obtained that the amygdala is a chemoreceptor that detects hypercarbia. ${ }^{3}$

Third, this work supplements knowledge of proximal neurobiological mechanisms with ideas about distal evolutionary mechanisms. Klein's paper arguing for the existence of an adaptive suffocation alarm that is falsely triggered in panic disorder is an extraordinary integration of a wide range of findings from disparate fields (e.g., the facts that patients with congenital central hypoventilation respond to panicogens, that patients with COPD have increased rates of panic disorder, and that panic attacks decrease during pregnancy).

Don was a man who was not shy to be critical, intending that such contributions would help progress the field. In that spirit, a number of limitations of work on the respiratory subtype deserve consideration.

Correspondence: Dan J. Stein, UCT Department of Psychiatry, Groote Schuur Hospital J2, Anzio Road, Observatory, Cape Town, 7925, South Africa.

E-mail: dan.stein@uct.ac.za

Submitted Apr 26 2020, accepted Apr 29 2020, Epub Jul 032020.
First, the clinical utility of the respiratory subtype seems limited. In particular, although there has been preliminary investigation of differential treatment response for the subtype, the evidence has not been considered sufficiently compelling to impact treatment guidelines. It is notable that neither DSM-5 nor ICD-11 have included this subtype as a specifier for panic disorder. Sadly, this may in turn inhibit investigation; a uniform way of defining the respiratory subtype would benefit further research.

Second, despite the elegance of the neurobiological and evolutionary accounts of the mechanisms underlying respiratory panic attacks, it seems that these mechanisms are neither necessary nor sufficient for the development of panic disorder. Instead, it seems that multiple different psychobiological pathways may play a role in panic disorder. ${ }^{4}$ The structure of mental disorders seems to be enormously complex; the precision of the respiratory subtype model needs to be matched with that of additional models in order to fully explain panic disorder.

Third, although past work was of a very high standard for its time, it is open to criticism from the perspective of contemporary approaches to addressing the replication crisis in psychology and medicine. ${ }^{5}$ In the future there will need to be more attention to issues such as preregistration of statistical analyses, sufficiently powered sample sizes, and open sharing of raw data. These kinds of strategies may help to resolve some of the contradictory findings in the field.

What is the best way to build on the foundations laid by Klein, Nardi, and Gorman? There are several possibilities; I will mention three.

A first idea is to substantially increase sample sizes of panic disorder patients, so allowing robustly powered and unbiased neuroimaging and neurogenetic analyses. Efforts are ongoing in this direction, by collaborations such as the Psychiatric Genetics Consortium and the Enhancing Imaging-Genetics by Meta-Analysis (ENIGMA) collaborations. Perhaps genome-wide association studies will yield polygenic risk score analyses, which, together with other measures, facilitate a personalized approach to treatment. That said, such efforts have not yet borne clinical fruit in psychiatry.

How to cite this article: Stein DJ. The respiratory subtype of panic disorder: reflections on the past and future of biological psychiatry. Braz J Psychiatry. 2020;42:340-341. http://dx.doi.org/10.1590/15164446-2020-1076 
A second idea is to employ translational and hypothesisfocused approaches (perhaps including the Research Domain Criteria framework), using current biological knowledge to develop and test medications that target the respiratory subtype. Previous trials of panic disorder have admitted all comers and have focused on symptom outcomes. An experimental medicine approach might study only patients with the respiratory subtype, with a focus on biological outcomes. Again, such a strategy has not yet proved successful in psychiatry, and given the complexity of causal pathways in mental disorders, success seems difficult to guarantee.

A third idea is to focus less on discovery research and more on implementation research. A cursory examination of the diagnosis and treatment of common mental disorders around the globe, and the resources available for this, reveals that an immediate priority is to develop efficacious treatments that can be delivered by community health workers, perhaps employing digital technologies. ${ }^{6}$ Global mental health has paid relatively little attention to anxiety disorders to date, and this seems an important gap.

While we are currently perhaps in a position to see further than Klein, Nardi, and Gorman by standing on their shoulders, it is difficult to see how future work will be as integrative and elegant as the first generation of work on the respiratory subtypes of panic disorder has been. Klein put great stock in careful observations by clinicians and rigorous clinical trials; today, the money is on big data analyses and trials informed by translational research.
Perhaps drawing on the best aspects of each of these approaches will allow the next generation of researchers to match the progress made by their predecessors.

\section{Acknowledgements}

The author is funded by the South African Medical Research Council.

\section{Disclosure}

The author has received research grants and/or consultancy honoraria from Lundbeck and Sun.

\section{References}

1 Okuro RT, Freire RC, Zin WA, Quagliato LA, Nardi AE. Panic disorder respiratory subtype: psychopathology and challenge tests - an update. Braz J Psychiatry. 2020;42:420-30.

2 Gorman JM, Liebowitz MR, Fyer AJ, Stein J. A neuroanatomical hypothesis for panic disorder. Am J Psychiatry. 1989;146:148-61.

3 Ziemann AE, Allen JE, Dahdaleh NS, Drebot II, Coryell MW, Wunsch $\mathrm{AM}$, et al. The amygdala is a chemosensor that detects carbon dioxide and acidosis to elicit fear behavior. Cell. 2009;139:1012-21.

4 Sobanski T, Wagner G. Functional neuroanatomy in panic disorder: status quo of the research. World J Psychiatry. 2017;7:12-33.

5 Munafò MR, Nosek BA, Bishop DVM, Button KS, Chambers CD, du Sert NP, et al. A manifesto for reproducible science. Nat Hum Behav. 2017;1:0021.

6 Patel V, Saxena S, Lund C, Thornicroft G, Baingana F, Bolton P, et al. The Lancet Commission on global mental health and sustainable development. Lancet. 2018;392:1553-98. 